\title{
An adaptive seamless assist-as-needed control scheme for lower extremity rehabilitation robots
}

\author{
Xiaodong Zhang ${ }^{1,2,3}$, Gui Yin ${ }^{1,2}{ }^{D}$, Hanzhe $\mathrm{Li}^{1,2}$, \\ Runlin Dong ${ }^{1,2}$ and Huosheng $\mathrm{Hu}^{3}$
}

\begin{abstract}
Most control methods deployed in lower extremity rehabilitation robots cannot automatically adjust to different gait cycle stages and different rehabilitation training modes for different impairment subjects. This article presents a continuous seamless assist-as-needed control method based on sliding mode adaptive control. A forgetting factor is introduced, and a small trajectory deviation from reference normal gait trajectory is used to learn the rehabilitation level of a human subject in real time. The assistance torque needed to complete the reference normal gait trajectory is learned through radial basis function neural networks, so that the rehabilitation robot can adaptively provide the assistance torque according to subject's needs. The performance and efficiency of this adaptive seamless assist-as-needed control scheme are tested and validated by 12 volunteers on a rehabilitation robot prototype. The results show that the proposed control scheme could adaptively reduce the robotic assistance according to subject's rehabilitation level, and the robotic assistance torque depends on the forgetting factor and the active participation level of subjects.
\end{abstract}

\section{Keywords}

Assist-as-needed control, lower extremity rehabilitation robot, radial basis function neural networks, forgetting factor, trajectory deviation

\section{Introduction}

Weight-supported treadmill training is an important way for the rehabilitation of patients with lower limb dyskinesia, and its effectiveness has been verified by a large number of clinical studies. ${ }^{1}$ Since patients have autonomy awareness, an interactive control between a rehabilitation robot and a patient is indispensable.,3 There are many human-robot interaction control strategies for rehabilitation robots in the world; for example, active leg exoskeleton $(\mathrm{ALEX})^{4,5}$ uses a force control scheme in the gait training for patients. However, the robotic assistance cannot be automatically adjusted by the interactive control method., Zhang et al. ${ }^{8}$ have designed a mixed position controller for an exoskeleton rehabilitation robot, in which, however, an assist-as-needed (AAN) assistance torque cannot be achieved by rehabilitation robots as patient's rehabilitation level is not taken into consideration.

It is hypothesized that patient's active participation in gait rehabilitation training may improve the rehabilitation outcomes. Up to now, AAN control framework is used to enhance patients' recovery through an activeassist training ${ }^{9-11}$ and impedance control is commonly used as an AAN gait rehabilitation training strategy. ${ }^{12-15}$ Toshio and Yoshiyuki ${ }^{16}$ performed manual tracking control tests on a human-robot system using an impedancecontrolled robot, and investigated control characteristics of a human operator according to robotic impedance properties. He and Dong ${ }^{17}$ proposed an adaptive fuzzy neural network control for a constrained robot using impedance learning. Pérez-Ibarra et al. ${ }^{18}$ proposed an AAN method for ankle rehabilitation based on adaptive

\footnotetext{
'School of Mechanical Engineering, Xi'an Jiaotong University, Xi'an, P.R. China

${ }^{2}$ Shaanxi Key Laboratory of Intelligent Robots, Xi'an Jiaotong University, Xi'an, P.R. China

${ }^{3}$ School of Computer Science and Electronics Engineering, University of Essex, Colchester, UK
}

\section{Corresponding author:}

Gui Yin, School of Mechanical Engineering, Xi'an Jiaotong University, Xi'an 710049, Shaanxi Province, P.R. China.

Email: 792000832@qq.com 
impedance control strategies, and the concept of AAN gait training was also considered for lower extremity powered exoskeleton (LOPES) ${ }^{19,20}$ Although the AAN control is achieved to some extent, they still need to manually switch between different modes and are unable to achieve adaptive AAN assistance torque. Chen et al. ${ }^{21}$ proposed an adaptive control strategy for gait rehabilitation robot to assist-when-needed. Cao et al. ${ }^{22}$ developed adaptive assistance control algorithms, which are also used to enhance patient's active participation. These methods cannot achieve seamless AAN gait training.

Recently, some scholars have used bioelectricity signals to achieve AAN control. Teramae et al. ${ }^{23}$ proposed a model predictive control based on Surface electromyography (sEMG) for AAN gait training. The AAN control was achieved using sEMG signal to estimate human torque directly, which was only for the upper limbs. Besides, Akim et al. ${ }^{24}$ proposed a braincomputer interface and AAN model for upper limb arm. Zhang et al. $^{25}$ designed a graph convolutional broad network (GCB-net) for emotion recognition. Zelinsky $^{26}$ developed a robot suit of hybrid assistive limb based on the bioelectricity signal. Wolbrecht et al. ${ }^{27}$ created optimizing compliant and model-based robotic assistance to promote neurorehabilitation, which was implemented in the task space of upper extremity rehabilitation robot. However, the AAN control for the lower extremity rehabilitation robot has not been developed and reported.

In addition, Hussain and $\mathrm{Xie}^{28}$ designed an intrinsically compliant robotic orthosis for gait rehabilitation in which an AAN control method and pneumatic flexible actuator were adopted. However, its seamless AAN control did not consider the influence of the inertial under the high-speed condition. $\mathrm{Wu}$ et al. ${ }^{29}$ proposed a minimalintervention-based admittance control strategy to induce the active participation of patients and maximize the use of recovered motor functions during training. The controller parameters should be adjusted in accordance with the hemiplegia degree of patients. Although the method can realize AAN control, it cannot be applied to different training modes in a seamless manner. Furthermore, the method is only proposed for upper extremity rehabilitation exoskeleton. In this study, we propose to use a sliding mode adaptive control (SMAC) framework to control a lower extremity rehabilitation robot system for continuous seamless AAN gait training. The developed rehabilitation robot can adaptively adjust to different gait cycle stages for subjects at different rehabilitation levels. It can maximize the participation of subjects in the gait rehabilitation training. Our method can first obtain the necessary torques from a combined human-robot dynamic model to guide subjects to complete a reference normal gait angle trajectory.

Based on the SMAC, a forgetting factor is introduced in our approach. Then, subjects return to the normal gait angle trajectory, and the angle deviation from the reference normal gait angle trajectory can reflect subject's active motion ability and effort based on the combined human-robot dynamic model. The assistance torque needed to complete the reference normal gait angle is learned through radial basis function (RBF) neural network in real time. Thus, adaptive assistance control is achieved according to subject's rehabilitation level and the seamless adaptive assistance torque is provided to the rehabilitation robot. The position controller is used to deal with the robotic model uncertainties. $^{30}$ Liu and his colleagues ${ }^{31,32}$ proposed a neural-network-based sliding mode control of an uncertain robot using dynamic model approximated switching gain. Yin et al. ${ }^{33,34}$ used robust control of underactuated systems to tackle the optimal control for uncertainty cases. While the robustness of SMAC was used in this article, the proposed AAN control can learn subject's active torque through a small trajectory error. Thus, the robotic assistance torque can be decreased with the subject's rehabilitation level and active participation level in the rehabilitation training, so that it can adaptively achieve AAN control. The active effort of subjects can be regarded as a disturbance. The robot can learn subject's rehabilitation level through a small trajectory deviation and adapt to subject's active effort in a seamless manner. There is no need to change parameters in different rehabilitation training stages, so that the control method could be applied to both the passive and active rehabilitation training modes.

Based on the robustness of SMAC, the trajectory deviation can be set very small. Since it used continuous gait angle trajectory deviation data to learn subject's active effort, there is no need to divide the support phase control and the swing phase control for this control method. It could be continuously and seamlessly adapted to different phases of the gait cycle. In addition, the performance of rehabilitation robots could be also validated among subjects with different rehabilitation levels. The rest of this article is organized as follows. The "Materials and methods" section introduces a lower extremity rehabilitation robot and its dynamic model as well as an adaptive continuous seamless AAN controller. Experiment setup is detailed in the "Experimental investigation" section, and results are given in the "Results and discussion" section to show the feasibility and performance of the proposed approach. Finally, a brief conclusion and future work are presented in the "Conclusion" section. This control method does not need to divide the support phase control and the swing phase control for separate control.

\section{Materials and methods}

\section{Design and modeling of lower extremity rehabilitation robot}

The lower limb exoskeleton rehabilitation robot completes the entire desired normal gait trajectory through the cooperation of human and robot. The lower limb rehabilitation robot assists the subjects in completing 


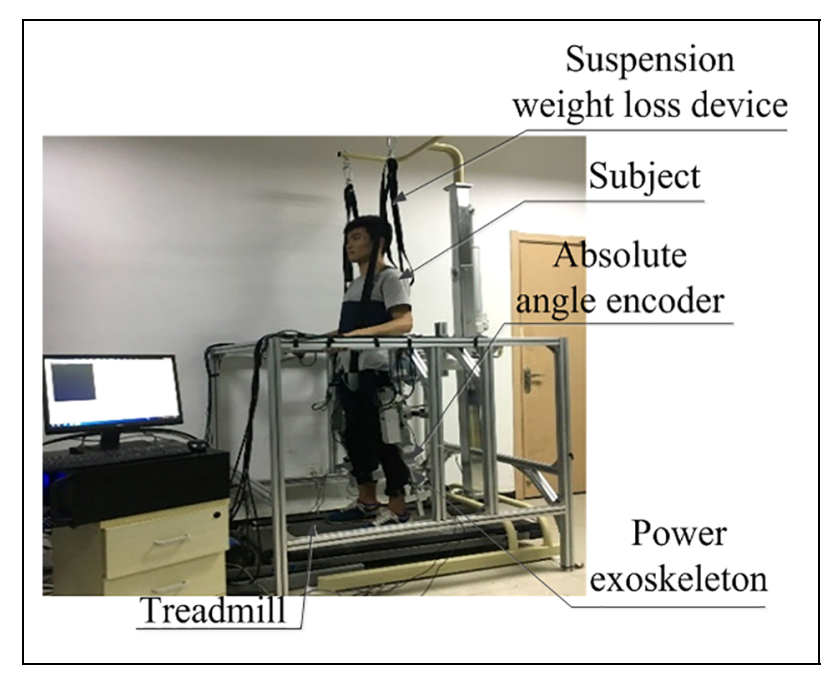

Figure I. The experimental setup of entire human-robot system for gait rehabilitation training.

the desired trajectory tracking. The entire human-robot system is shown in Figure 1.

As is shown in Figure 1, the lower extremity rehabilitation training robot is powered by a crank slider mechanism, and it is developed using the biometric design method in our laboratory. The rehabilitation robot consists of a power exoskeleton, treadmill, suspension weight loss device, and other components; the lengths of exoskeleton thigh and calf sections are adjustable to fit into patients with different heights from 1.56 to $1.81 \mathrm{~m}$; and the Intrepid MTC-2.2A treadmill is used and integrated with exoskeleton.

Figure 2 shows the power exoskeleton that is the main part of rehabilitation robot, in which the flexion and extension movement for the hip and knee joints is the actuated degree-of-freedom (DOF) of the exoskeleton. Its drive part deploys a crank slider mechanism in which the servo motor is the active element to drive the ball screw nut moving in a straight line along the screw rod. The straight-line movement of the nut is changed into the rotation of the joint through the crank slider mechanism. To accommodate patients with different heights, the inner tube of the thigh and calf rods can be moved along the outer tube to the adjusted length and fixed with a quick clamping device. Two holders are installed on the thigh and the calf of the exoskeleton, and their mounting locations are adjustable. In addition, a round hole and a fixed pressure plate are arranged at hip and knee joints for the encoder installation. Note that the complete design description of the rehabilitation robot can be found in the article. ${ }^{35}$

There are two absolute angle encoders in each power exoskeleton device, which are used to measure robotic joint angle in real time. The encode data are sent to the robotic controller for calculating robotic trajectory deviation relative to the reference normal gait angle trajectory. Note that the absolute encoder has a 14-bit resolution and high-speed digital communication output

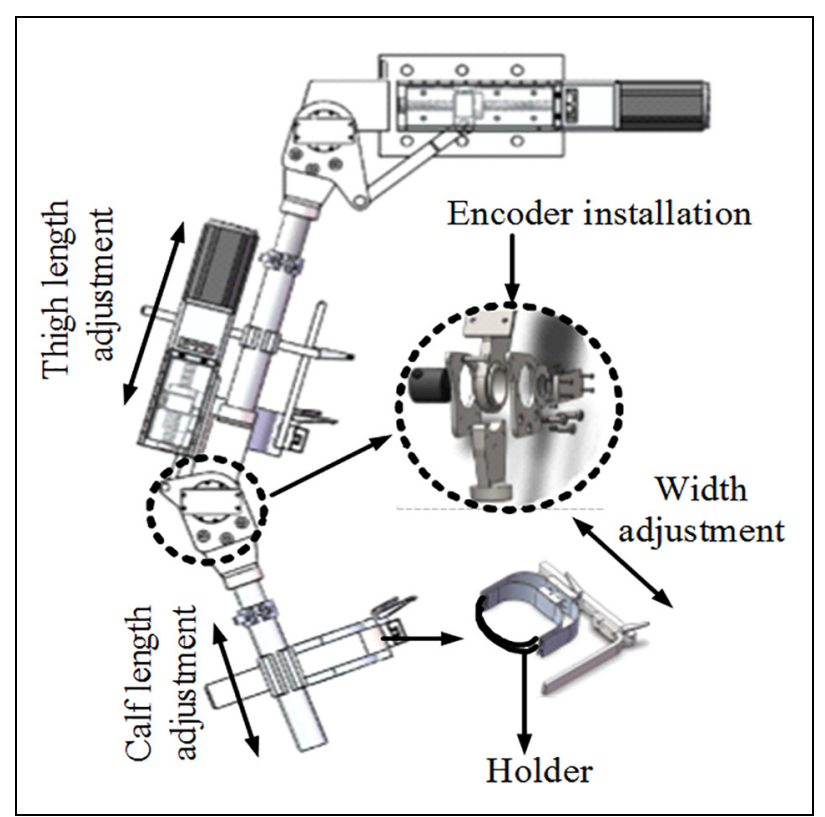

Figure 2. The configuration of power exoskeleton.

so that it can realize high precision digital control. A single power exoskeleton is equivalent to a two-bar linkage and closely attached to human leg; the joint angle errors between human and robot are relatively small. ${ }^{36}$ Assuming that subject's movement and the robotic movement are synchronized below

$$
\theta=\theta_{h}
$$

where $\theta_{h}$ and $\theta$ represent human angle and robotic angle, respectively.

When the robot dynamic model is specified by the Lagrange dynamic equation, the combined dynamics of the human-robot system can be given below ${ }^{37}$

$$
M(\theta) \ddot{\theta}+C(\theta, \dot{\theta}) \dot{\theta}+G(\theta)=T_{r o b}+T_{h}
$$

where $\theta, \dot{\theta}$, and $\ddot{\theta}$ represent $2 \times 1$ vectors of the generalized angle, angular velocity, and angular acceleration for the human-robot system, respectively. $M(\theta)$ is the generalized $2 \times 2$ inertial force matrix. $C(\theta, \dot{\theta})$ denotes the $2 \times 2$ centrifugal and Coriolis matrices. $G(\theta)$ is the $2 \times 1$ vector of gravitational torque. $T_{r o b}$ represents $2 \times 1$ vector of the robotic torque, and $T_{h}$ represents $2 \times 1$ vector of the active torque of subject.

\section{Adaptive continuous seamless AAN controller design for lower extremity rehabilitation robot}

When immobility resulted from muscle atrophy or other reasons, subjects should undergo passive rehabilitation training. As their rehabilitation level increases, subjects would be motivated to participate in gait rehabilitation training and robotic assistance torque needs to reduce in real time. Then, a forgetting factor $\tau$ is introduced based on SMAC. The partial derivative of 


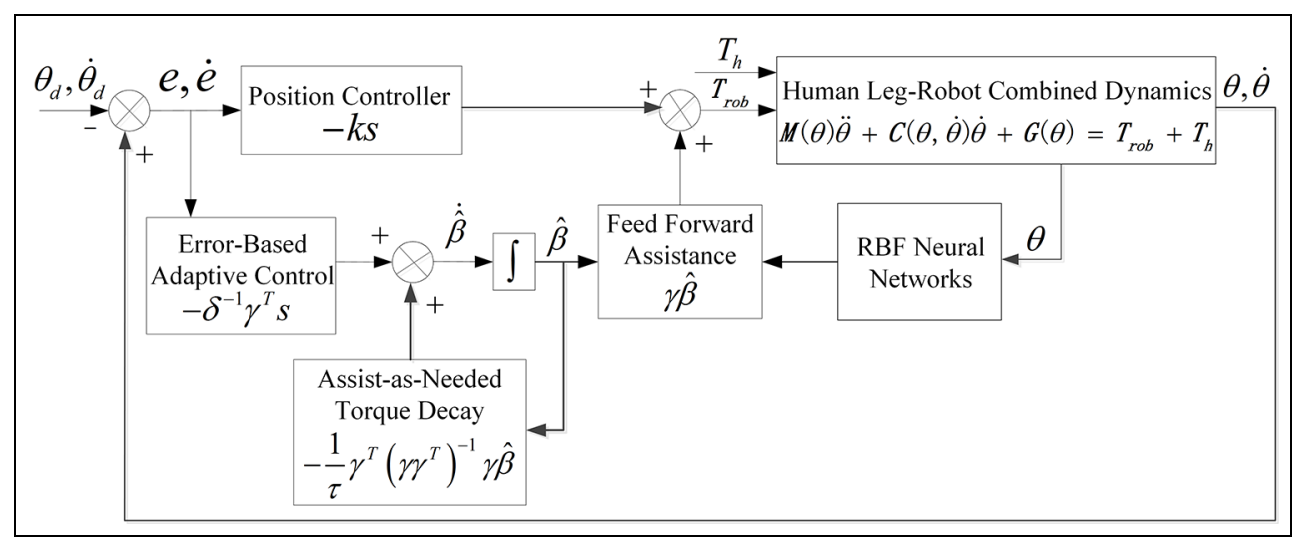

Figure 3. The adaptive seamless AAN control diagram for lower extremity rehabilitation robot.

the feed forward assistance $\gamma \hat{\beta}$ is used to limit the change in $\hat{\beta}$, which allows the controller to learn the subject's active torque as a general function of reference normal gait angle, angular velocity and angular acceleration of subject, as shown in Figure 3. The active effort of subject can be regarded as a disturbance and the robot can learn subject's rehabilitation level through a small trajectory deviation and adapt to subject's active effort in a seamless manner. There is no need to change parameters in different rehabilitation stages.

The RBF neural network has the adaptive function of self-learning and self-organization. Moreover, the connection weight of RBF neural network is linear with its output, and the weight is the coefficient of the amount of the assistance torque needed to complete the reference normal gait trajectory. It is convenient to use the Gaussian function to form the regression matrix, and the linear multiplication with the weights constitutes the feed forward assistance torque, which has sufficient resolution to the active torque of subject. Therefore, the feed forward assistance torque is learned from the RBF neural network to provide the seamless adaptive assistance torque for rehabilitation robots.

As shown in Figure 3, the overall structure of adaptive seamless AAN control scheme for lower extremity rehabilitation robot is based on an SMAC framework. $^{38,39}$ The sliding surface $s$ and the virtual reference trajectory $v$ for the adaptive seamless AAN controller are defined as follows

$$
\begin{aligned}
& s=\dot{e}+\alpha e \\
& v=\theta_{d}-\alpha e \\
& e=\theta-\theta_{d}
\end{aligned}
$$

where $e$ represents the robotic trajectory error for hip or knee joint. $\theta_{d}$ and $\theta$ represent the desired normal angle and actual angle for robotic hip or knee joint, respectively. $\alpha$ represents the positive design parameters.

The adaptive control law for the desired torque of rehabilitation robot can be expressed as follows

$$
T_{r o b}=\gamma(\theta, \dot{\theta}, v, \dot{v}) \hat{\beta}-k s
$$

where $\gamma \hat{\beta}$ is a combined model of robotic exoskeleton and human leg, including the active torque of subject during gait training, which is defined below

$$
\gamma \hat{\beta}=\hat{M} \ddot{v}+\hat{C} \dot{v}+\hat{G}-\hat{T}_{h}
$$

where $\hat{M}$ is the estimated inertial force matrix, $\hat{C}$ is the estimated centripetal and Coriolis matrix, and $\hat{G}$ is the estimated gravitational torques for combined humanrobot system. $\ddot{v}$ and $\dot{v}$ represent the acceleration and velocity of virtual trajectory, respectively. $\hat{T}_{h}$ represents subject's estimated active torque.

The feed forward assistance torque, including subject's active torque, should adapt to different subjects with different rehabilitation levels. In this article, the active torque of subject is modeled by the Gaussian RBFs, ${ }^{28}$ which is defined below

$$
g=\exp \left(-\frac{|\theta-\mu|^{2}}{2 \sigma^{2}}\right)
$$

where $g$ is the Gaussian RBFs for the subject's hip joint or knee joint. $\theta$ represents the current angle for the subject's hip joint or knee joint. $\mu$ denotes the center of $\mathrm{RBF}$, and $\sigma$ is the width of RBF.

According to the optimal results of subject's active torque estimation, the Gaussian RBF is divided into seven nodes, and the grid divisions are equally spaced at 10 degrees. In addition, the width of the Gaussian $\mathrm{RBF}$ is 5.81 degrees. The vector of Gaussian RBFs is defined as

$$
g=\left[\begin{array}{lllllll}
g_{1} & g_{2} & g_{3} & g_{4} & g_{5} & g_{6} & g_{7}
\end{array}\right]^{T}
$$

Since there are two different joints of human lower limb in this study, the regression matrix, $\gamma$, can be defined below according to ${ }^{27}$

$$
\gamma^{2 \times 14}=\left[\begin{array}{cc}
g^{T} & 0 \\
0 & g^{T}
\end{array}\right]
$$


Thus, the estimated weight of RBF neural network, $\hat{\beta}$, is a $14 \times 1$ vector, and the parameter represents the amount of assistance torque needed to complete the desired normal gait trajectory. The parameter vector is updated over time, and the adaptive law of the parameter is expressed as follows

$$
\dot{\hat{\beta}}=-\frac{1}{\tau} \gamma^{T}\left(\gamma \gamma^{T}\right)^{-1} \gamma \hat{\beta}-\delta^{-1} \gamma^{T} S
$$

where $\dot{\hat{\beta}}$ is the parameter adaptation rate, $\delta$ determines the adaptation rate based on the trajectory error, and $\tau$ denotes the forgetting factor of the rehabilitation robot.

The first term of equation (11) is used to reduce the robotic feed forward assistance according to subject's motion ability and effort, and the torque output of rehabilitation robot is reduced through the forgetting factor $\tau$ to achieve adaptive seamless AAN control. The second term is used to reduce the trajectory error of human-robot system to deal with robotic model uncertainties and external disturbances. Due to human torque component, the overall control system is not globally asymptotically stable. However, the proposed control method is ultimately bounded.

The Lyapunov function candidate can be defined as follows

$$
V(t)=\frac{1}{2} s^{T} M s+e^{T} k \alpha e+\frac{1}{2} \tilde{\beta}^{T} \delta \tilde{\beta}
$$

where $M, s, k, \alpha, e$, and $\delta$ are defined in the previous sections and $\tilde{\beta}$ is defined below

$$
\tilde{\beta}=\hat{\beta}-\beta
$$

where $\tilde{\beta}$ represents the parameter estimate error and $\hat{\beta}$ is an estimation of the parameter $\beta$. Differentiating equation (12) yields

$$
\dot{V}(t)=s^{T} M \dot{s}+\frac{1}{2} s^{T} \dot{M} s+2 e^{T} k \alpha \dot{e}+\tilde{\beta}^{T} \delta \dot{\tilde{\beta}}
$$

The rehabilitation robot dynamics in equation (2) can be redefined by using the sliding surface $s$ in equation (3) and the virtual reference trajectory $v$ in equation (4)

$$
M \dot{s}+C s+\gamma \beta=T_{r o b}
$$
as

Combining equation (15), $\dot{V}$ can be further expressed

$$
\dot{V}(t)=s^{T}\left(T_{r o b}-C s-\gamma \beta\right)+\frac{1}{2} s^{T} \dot{M} s+2 e^{T} k \alpha \dot{e}+\tilde{\beta}^{T} \delta \dot{\tilde{\beta}}
$$

Recalling that ( $\beta$ constant over time) $\dot{\beta}=0$, combining equation (13), and the rehabilitation robot dynamic properties, $\dot{M}-2 C=0$, equation (16) becomes

$$
\dot{V}(t)=s^{T}\left(T_{r o b}-\gamma \hat{\beta}\right)+2 e^{T} k \alpha \dot{e}+\tilde{\beta}^{T}\left(\gamma^{T} s+\delta \dot{\hat{\beta}}\right)
$$

Substituting $\gamma \hat{\beta}$ in equation (7), $\dot{\hat{\beta}}$ in equation (11), and $T_{r o b}$ in equation (6) yields

$$
\dot{V}(t)=-\dot{e}^{T} k \dot{e}-e^{T} k \alpha^{2} e-\tilde{\beta}^{T} \delta \gamma^{T}\left(\gamma \gamma^{T}\right)^{-1} \gamma \hat{\beta} / \tau
$$

Since the sign of $\tilde{\beta}^{T} \delta \gamma^{T}\left(\gamma \gamma^{T}\right)^{-1} \gamma \hat{\beta} / \tau$ can be either positive or negative, it is not zero and it cannot prove the negative definiteness of $\dot{V}$. However, we can define $E=\left[\begin{array}{ll}e & \dot{e}\end{array}\right]^{T}, A=\operatorname{diag}\left(k \alpha^{2}, k\right)$, and $D=\delta \gamma^{T}\left(\gamma \gamma^{T}\right)^{-1}$ $\gamma / \tau$. Thus, equation (18) can be rewritten as

$$
\dot{V}(t)=-E^{T} A E-\tilde{\beta}^{T} D \hat{\beta}
$$

If $E^{T} A E>-\tilde{\beta}^{T} D \hat{\beta}$, then $\dot{V}(t)<0$. A sufficient condition is

$$
E^{T} \alpha_{\min }(A) E>\max _{\hat{\beta}}\left(\tilde{\beta}^{T} D \hat{\beta}\right)
$$

where $\alpha_{\min }(A)$ is the minimum eigenvalue of $A$ and $\max _{\hat{\beta}}\left(\tilde{\beta}^{T} D \hat{\beta}\right)$ represents the maximum of $\tilde{\beta}^{T} D \hat{\beta}$, which occurs when $\hat{\beta}=\beta / 2$. Thus, the sufficient condition for $\dot{V}(t)<0$ is

$$
E>\frac{1}{2}\left(\frac{\beta^{T} D \hat{\beta}}{\alpha_{\min }(A)}\right)^{1 / 2}
$$

Equation (21) represents the set of $E$, in which the Lyapunov function is decreasing over time. It is to conclude that $e$ converges to the complement of equation (21), which is defined as

$$
|e| \leqslant \frac{1}{2}\left(\frac{\beta^{T} D \beta}{\alpha_{\min }(A)}\right)^{1 / 2}
$$

Recalling the expression of $D$ and the fact that $\alpha_{\max }\left(\delta \gamma^{T}\left(\gamma \gamma^{T}\right)^{-1} \gamma\right)=\alpha_{\max }(\delta)$, the ultimate boundary of $e$ is expressed as follows

$$
|e| \leqslant \frac{1}{2} \beta\left(\frac{1}{\tau} \frac{\alpha_{\max }(\delta)}{\alpha_{\min }(A)}\right)^{1 / 2}
$$

This shows that the tracking error of human-robot system will converge to the boundary. In addition, this upper bound is proportional to the Euclidean norm of vector $\beta$ and the forgetting term $1 / \tau$ of the proposed continuous seamless adaptive AAN controller.

\section{Experimental investigation}

\section{Experimental setup}

To verify the effectiveness of the proposed continuous seamless adaptive AAN control method, we select 12 subjects with different lower extremity movement impairments (6 male and 6 female, height $1.58-1.81 \mathrm{~m}$ (mean $1.698 \mathrm{~m}$ and standard deviation $0.051 \mathrm{~m}$ ), weight $50.1-73.8 \mathrm{~kg}$ (mean $61.6 \mathrm{~kg}$ and standard deviation $5.391 \mathrm{~kg}$ )). The different heights will not influence the control strategy and parameters of rehabilitation robot, and the rehabilitation robot prototype shown in Figure 1 is deployed in experiments. All of procedures have been approved by the Institutional Ethical Committee. All of the subjects' rehabilitation levels are assessed by muscle strength due to different muscle 
recovery conditions. According to the Kendall classification principle for muscle strength assessment, the knee joint torque generated by the muscle contraction of normal human at a normal walking speed $(1 \mathrm{~m} / \mathrm{s})$ is used as the standard reference torque for complete rehabilitation.

Then the standard reference torque is used to assess different rehabilitation levels of subjects: mild rehabilitation $(20 \%$ of the standard reference torque and a deviation of $3 \mathrm{Nm})$, moderate rehabilitation $(50 \%$ of the standard reference torque and a deviation of $3 \mathrm{Nm})$, and basic rehabilitation $(85 \%$ of the standard reference torque and a deviation of $3 \mathrm{Nm}$ ). There are four subjects (two male and two female) in each rehabilitation level for the experimental validation. In the control experiments, the sample frequency is $1 \mathrm{kHz}$, and a third-order Butterworth filter is used to filter the collected angle and torque data. Human walking speed is $1.0 \mathrm{~m} / \mathrm{s}$. According to the optimal control results, the controller parameters are selected: $\alpha=10 I_{2 \times 2}$, $\delta=0.15 I_{14 \times 14}, k=\operatorname{diag}(84,64)$. The gait cycle time is set to $1.2 \mathrm{~s}$, the forgetting rate $\tau$ was chosen by trial and error during the experiments, and the maximum trajectory error from the reference normal gait angle is set to 3 degrees to determine the value of the forgetting factor $\tau$. Then, the experiments are performed with different subjects.

\section{Experimental procedure}

The gait trajectory tracking experiment and AAN adaptive experiment are performed with subjects. The gait trajectory tracking control experiment aims to validate whether the rehabilitation robot is capable of assisting subjects at all various rehabilitation levels to reach the normal gait angle range, and whether the rehabilitation robot could guide subjects' limbs on the reference normal gait angle when the forgetting factor is introduced. The AAN adaptive experiment aims to test whether the AAN controller could learn the torque needed to complete the desired normal gait angle and allow the subject to participate in gait rehabilitation training as actively as possible to improve the gait rehabilitation effect.

The gait trajectory tracking experiment is conducted for two modes: without assistance mode and with assistance mode. First, the subjects with different rehabilitation levels attempted to track a normal gait angle range without robotic assistance. In this trial, the effect of the rehabilitation robot is excluded, and the net assistance torque on the subjects is approximately zero. For the second mode, the rehabilitation robot assists the subjects to track the normal gait angle range. During the experiment, all the subjects should track the gait angle ranges three times. Then they are passive and their gait angle trajectories are guided by the rehabilitation robot. The value of forgetting factor $\tau$ should be determined by the maximum trajectory deviation from the reference normal gait angle.

Specifically, the forgetting factor value should be gradually decreased until the AAN controller would no longer guide subjects' limbs to reach the desired normal gait trajectories. The forgetting factor value should still be used for the AAN adaptive experiment. The AAN adaptive experiment should be conducted at always active and passive-to-active modes. For the always active mode, subjects are instructed to actively track the desired normal gait trajectories for 50 gait cycles. In the passive-to-active mode, subjects remain passive and their gait trajectories are guided by the rehabilitation robot during the first 20 gait cycles. The controller will learn the needed torques to complete the reference normal gait angles. Then, subjects are instructed to actively track the desired normal gait trajectories for the last 30 gait cycles. The aim of this mode is to determine whether the AAN controller could reduce the robotic torque to increase subjects' active voluntary torque output.

\section{Results and discussion}

\section{Gait trajectory tracking experimental results}

Due to individual differences, there is a deviation for the upper bound or the lower bound of the gait angle ranges. There is an uncertainty in the lower or upper bounds of the parameters as described in the literature. $^{40,41}$ Compared with the literature, ${ }^{40,41}$ the gait angle range for the subject at different rehabilitation level is fuzzy, but the range of the upper bound or the lower bound is defined. We can define the lower and upper bounds of the angle range, which are the "crisp values" of the fuzzy variable. In fact, the fuzzy bound can be interpreted as the description of "close to or around the crisp value." Under this situation, the uncertainty of angle range can be represented by a standard deviation of the bound for a dual fuzzy boundary: bound crisp value (standard deviation). Table 1 presents the gait angle ranges of different subjects for different modes during the gait trajectory tracking experiment.

As can be seen, without assistance, subjects with mild rehabilitation and moderate rehabilitation levels cannot reach the normal lower bound and upper bound, while subjects with basic rehabilitation levels can almost reach the normal lower bound and upper bound. However, with assistance, the rehabilitation robot successfully assisted all the subjects at any rehabilitation level in performing gait trajectory tracking to reach the normal gait angle range. Indeed, the rehabilitation robot controller allowed all the subjects to reach the reference normal gait angle targets successfully.

The forgetting factor value determined by the trajectory tracking experiment is 0.5 . Trajectory tracking 
Table I. Subject's gait angle ranges.

\begin{tabular}{llllll}
\hline \multirow{2}{*}{ Subjects } & \multicolumn{2}{l}{ Without assistance } & & \multicolumn{2}{l}{ With assistance } \\
\cline { 2 - 3 } \cline { 5 - 6 } & Lower bound & Upper bound & & Lower bound & Upper bound \\
\hline Hip angle range of mild rehabilitation & $-6.13(0.24)^{\circ}$ & $6.08(0.13)^{\circ}$ & & $-21.05(0.26)^{\circ}$ & $21.06(0.19)^{\circ}$ \\
Knee angle range of mild rehabilitation & $0(0.18)^{\circ}$ & $20.16(0.15)^{\circ}$ & & $0(0.19)^{\circ}$ & $60.26(0.23)^{\circ}$ \\
Hip angle range of moderate rehabilitation & $-13.16(0.18)^{\circ}$ & $13.39(0.21)^{\circ}$ & & $-21.06(0.15)^{\circ}$ & $20.98(0.26)^{\circ}$ \\
Knee angle range of moderate rehabilitation & $0(0.22)^{\circ}$ & $40.18(0.21)^{\circ}$ & & $0(0.24)^{\circ}$ & $60.21(0.28)^{\circ}$ \\
Hip angle range of basic rehabilitation & $-19.98(0.27)^{\circ}$ & $20.03(0.28)^{\circ}$ & & $-21.09(0.24)^{\circ}$ & $21.07(0.26)^{\circ}$ \\
Knee angle range of basic rehabilitation & $0(0.23)^{\circ}$ & $60.01(0.25)^{\circ}$ & & $0(0.28)^{\circ}$ & $60.33(0.26)^{\circ}$ \\
\hline
\end{tabular}

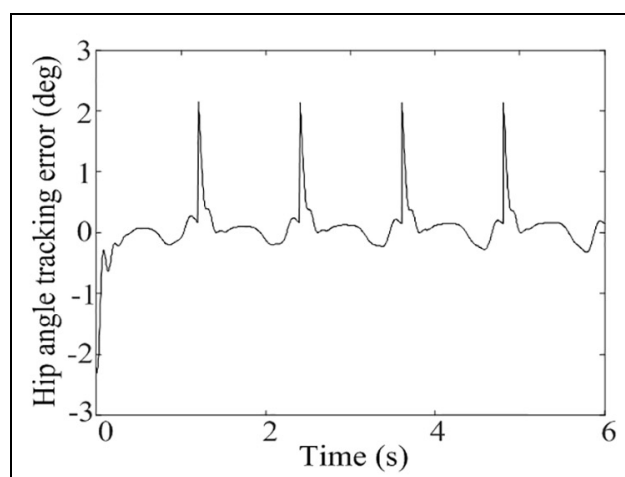

(a)

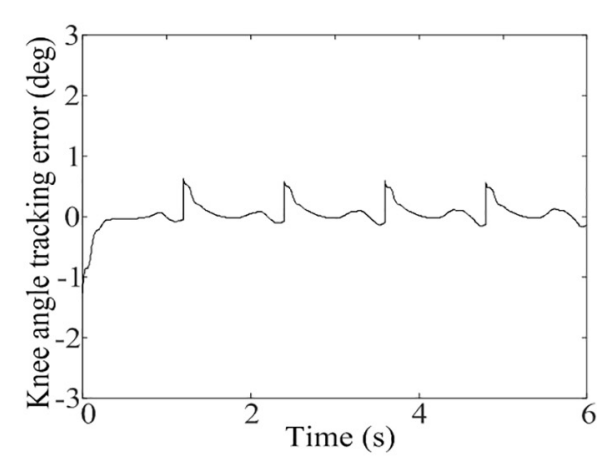

(b)

Figure 4. Robotic trajectory tracking errors without the forgetting term: (a) hip tracking error and (b) knee tracking error.

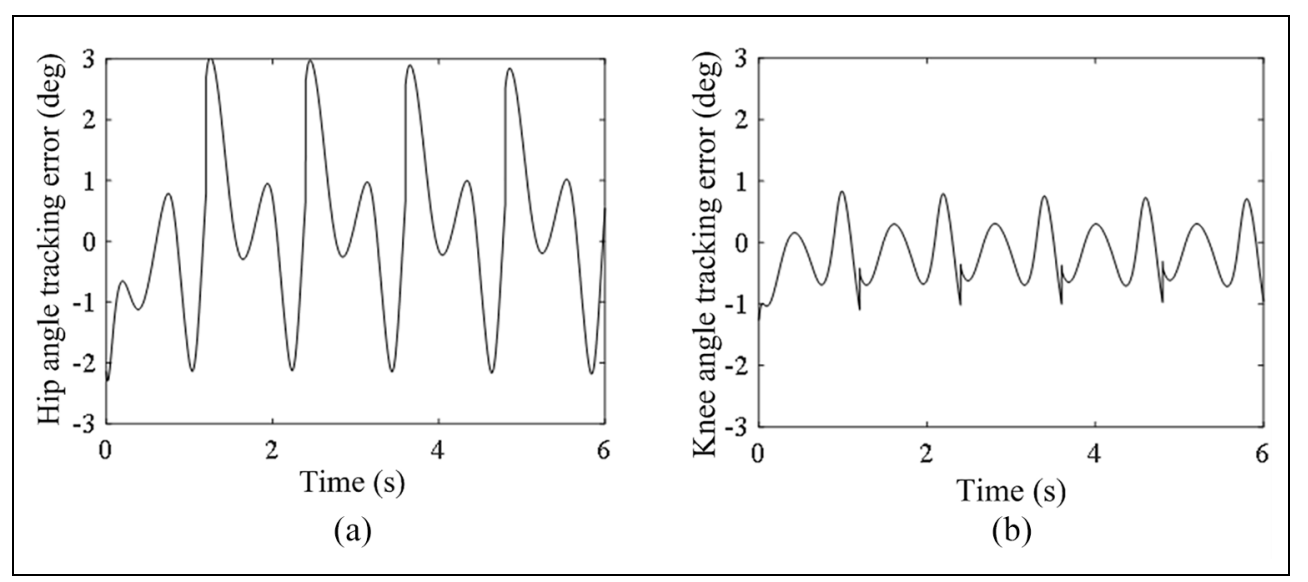

Figure 5. Robotic trajectory tracking errors with the forgetting term: (a) hip tracking error and (b) knee tracking error.

results without the forgetting term (the forgetting factor is infinite) for the normal human (complete rehabilitation) are given in Figure 4. For further comparison, the trajectory tracking results with the forgetting term are shown in Figure 5.

From the above results, the trajectory tracking errors (angle deviations from the reference normal gait trajectories) with the forgetting term are larger than those without the forgetting term. Moreover, with inclusion of the forgetting term, the maximum angle deviations of the hip and knee joints are less than 3 degrees; this is a high trajectory tracking accuracy. The maximum trajectory tracking error of LOKOMAT is up to 11 degrees during the gait rehabilitation training. ${ }^{42}$ However, the deviation from the desired normal gait angle trajectory is relatively small using our control method. It can meet the gait training needs for all the subjects at different rehabilitation levels. 


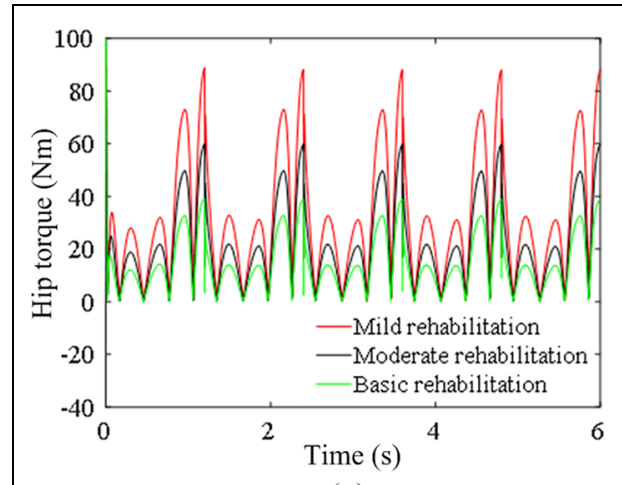

(a)

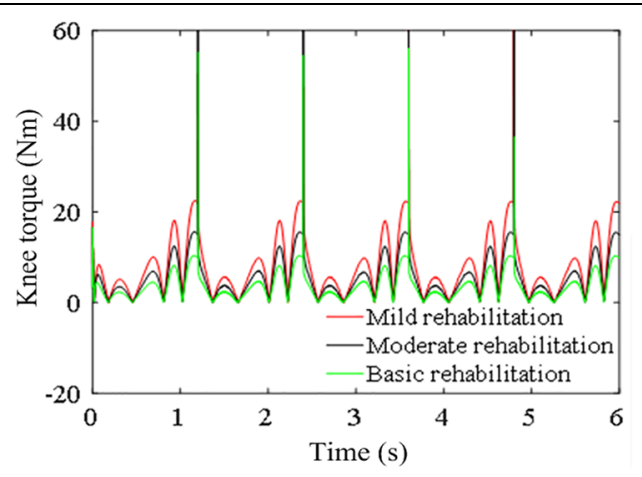

(b)

Figure 6. The mean of robotic joint assistance torques for subjects with different rehabilitation levels: (a) hip assistance torque and (b) knee assistance torque.

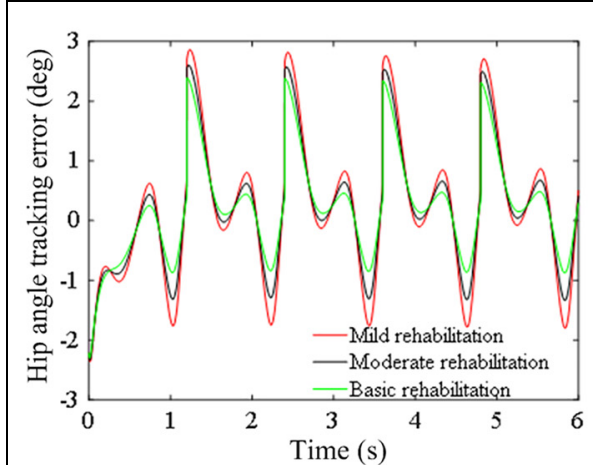

(a)

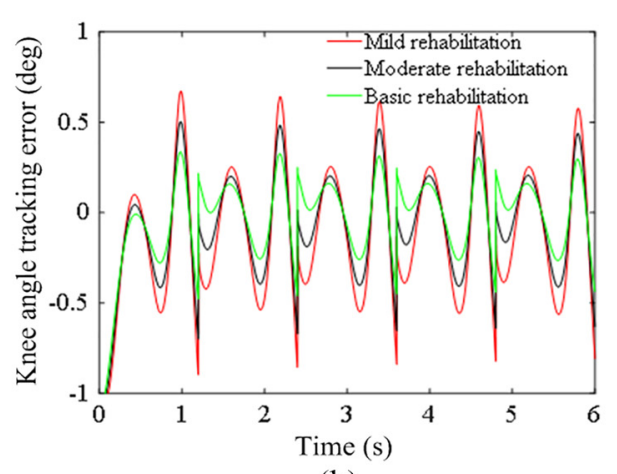

(b)

Figure 7. The mean of robotic trajectory tracking errors for subjects with different rehabilitation levels: (a) hip tracking error and (b) knee tracking error.

\section{AAN adaptive experimental results}

The AAN adaptive control experiments were also performed for two modes. The assistance torques of the rehabilitation robot during the always active mode, where the forgetting factor is 0.5 , for subjects with different rehabilitation levels are shown in Figure 6.

As Figure 6 shows, the AAN controller could decay the robotic assistance torque as the subject's rehabilitation level increases. It allows the subject to actively participate in the gait rehabilitation training procedure as much as possible to improve the subject's gait rehabilitation effect. It can be known from the existing literature that the human normal hip drive torque is approximately $80 \mathrm{Nm}$. However, the results show the hip assistance torque is greater than $80 \mathrm{Nm}$ when the subject is at a mild rehabilitation level. The experimental results are for the entire human-robot system, and the robotic assistance torque is also used to drive the rehabilitation robot itself to complete the reference normal gait angle. Therefore, the hip assistance torque may become greater than the driving torque required by the normal human individual movement. Moreover, the speed of the knee joint changes substantially during the gait cycle conversion.

It is known from the previous human-robot dynamic model that the effect of human plantar force and the effort of the treadmill are neglected. At the moment that the foot touches the ground, the effort of the treadmill will have an impulse on the knee joint, thereby resulting in the judder of the knee torque. However, in each gait cycle, the robotic assistance torque gradually decreases as the subject's rehabilitation level increases. The mean trajectory tracking errors of the rehabilitation robot for subjects with different rehabilitation levels during the always active mode with a forgetting factor of 0.5 are shown in Figure 7.

As can be seen in Figures 6 and 7, the AAN controller can learn the subject's rehabilitation level in real time via a small trajectory error in terms of the forgetting factor. Since the gait angle trajectory is continuous, the control method used a small trajectory error data to learn subject's active effort, and it is no need for swing 


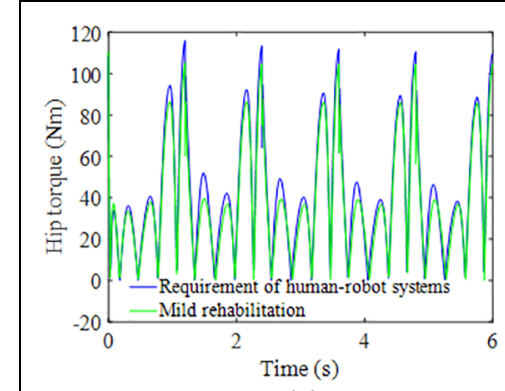

(a)

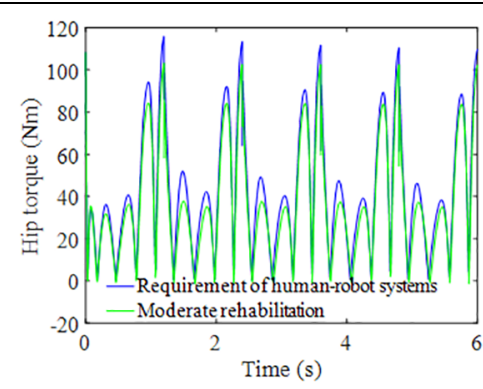

Time (s)

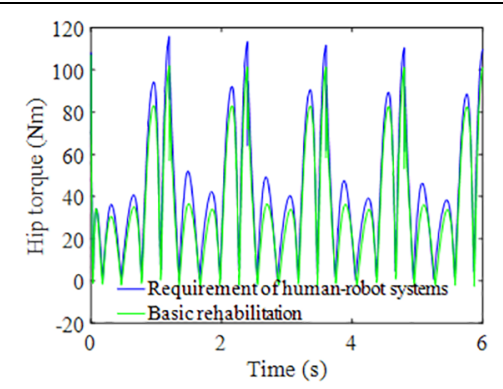

(c)

Figure 8. The mean of robotic hip assistance torques for subjects with different rehabilitation levels: (a) mild rehabilitation, (b) moderate rehabilitation, and (c) basic rehabilitation.

phase control and support phase control, separately. Thereby, it could be continuously adapted to different phases of the gait cycle. As the subject's rehabilitation level increases, the robotic assistance torque was gradually reduced to improve the subject's gait rehabilitation effect. Meanwhile, it can ensure a high trajectory tracking accuracy, which can meet the gait rehabilitation training requirement of subjects with different rehabilitation levels. Thus, this could be applied to both the passive and active rehabilitation training modes to achieve adaptive seamless AAN control. In the absence of the forgetting term, the assistance torques of the rehabilitation robot during the always active mode for subjects with different rehabilitation levels (comparative analysis of hip joints only) are shown in Figure 8.

As shown in Figure 8, it can be seen that when the forgetting term is not included, the robotic assistance torque is not reduced and remains at a high level regardless of subject's rehabilitation level. It is just a little less than the entire human-robot system needed, owing to the effect of forgetting term. It is shown that when the forgetting term is not used, the lower extremity rehabilitation robot replaces subject to complete the gait angle trajectory, which decreases the subject's active participation level and inhibits the active effort of subject. It is obvious that the assistance torque of rehabilitation robot is affected by both the forgetting factor value and the amount of active participation of subject. Finally, the steady-state value is reached.

Another rehabilitation mode is passive-to-active rehabilitation mode. The mean of robotic assistance torques for subjects with different rehabilitation levels during the passive-to-active mode (comparative analysis of hip joints only) is presented in Figure 9. As can be seen, the subjects perform passive gait rehabilitation training during the first two gait cycles, and the subjects perform active gait rehabilitation training during the last three gait cycles. When the forgetting factor is included and the subject is in active rehabilitation training, the hip maximum assistance torque of rehabilitation robot is $91 \mathrm{Nm}$ for subjects with a mild rehabilitation level. However, for subjects with a moderate rehabilitation level or basic rehabilitation level, the hip maximum assistance torque of rehabilitation robot is 60 and $39 \mathrm{Nm}$, respectively. It is obvious that when a forgetting factor is used, the robotic assistance torque gradually decreases as the subject's rehabilitation level increases. When the forgetting term is not included, and subjects transited from passive training to active training, the hip assistance torque of the rehabilitation robot remains at a high level and decreases slightly. Even if the subjects have active motion ability, they could not be made to obtain full usage.

Table 2 shows the maximum gait angle deviation from the desired normal gait trajectory for subjects with a basic rehabilitation level and the maximum assistance torque statistical results (only the data from the last three gait cycles) of the rehabilitation robot for different gait training modes. As can be seen, the hip maximum angle deviation with the forgetting term is larger than that without the forgetting term for both the always active mode and the passive-to-active mode. With the forgetting term, the hip maximum angle deviations have exceeded the set deviation for trajectory tracking. This is mainly due to the impact of the machining accuracy and assembly errors of the robotic transmission mechanism, and the delay of the robotic measurement and control system. Without the forgetting term, the hip maximum assistance torque of rehabilitation robot is very high. However, with the forgetting term, the hip maximum assistance torque of the rehabilitation robot decreases obviously, because the subject actively participates in the gait rehabilitation training. It is clear that with the forgetting term, the rehabilitation robot increases the trajectory error to learn subject's active torque and adaptively achieves AAN control.

In summary, with the forgetting term, the AAN controller can learn subject's active torque through a small trajectory error. Thus, the subject can actively participate in the rehabilitation training as much as possible to decrease the robotic assistance torque and adaptively achieve AAN control. The rehabilitation robot prototype experiments illustrate the feasibility of the proposed continuous seamless adaptive AAN control method. When the forgetting term is used, the adaptive AAN controller can effectively decrease robotic 


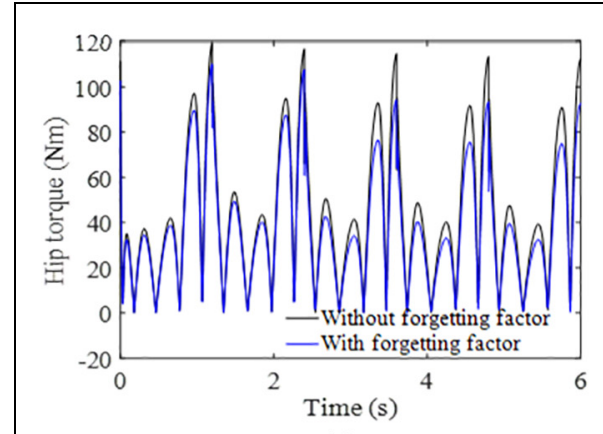

(a)

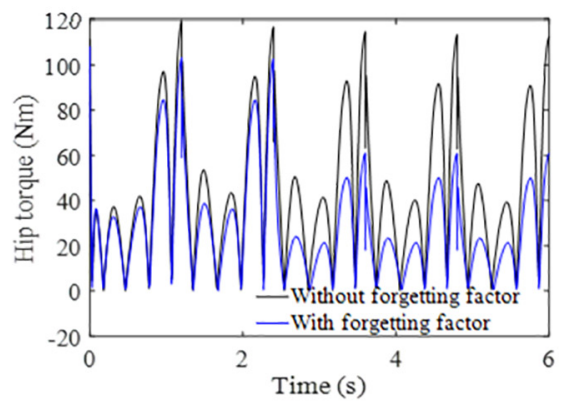

(b)

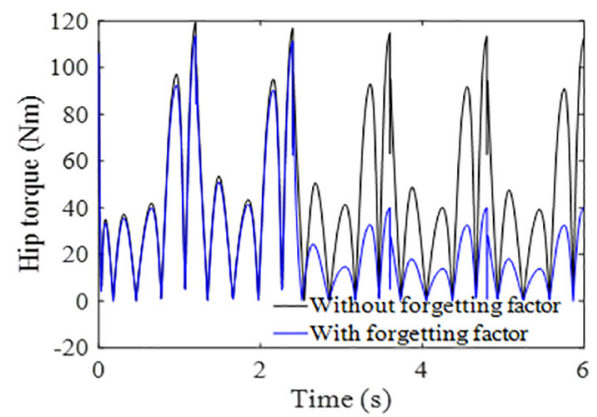

(c)

Figure 9. The mean of robotic hip assistance torques for subjects with different rehabilitation levels during the passive-to-active mode: (a) mild rehabilitation, (b) moderate rehabilitation, and (c) basic rehabilitation.

Table 2. Subject's maximum gait angle deviation and robotic maximum assistance torque.

\begin{tabular}{|c|c|c|c|c|}
\hline \multirow[t]{2}{*}{ Gait parameter } & \multicolumn{2}{|l|}{ Always active mode } & \multicolumn{2}{|l|}{ Passive-to-active mode } \\
\hline & $\begin{array}{l}\text { Without the forgetting } \\
\text { term }\end{array}$ & $\begin{array}{l}\text { With the forgetting } \\
\text { term }\end{array}$ & $\begin{array}{l}\text { Without the forgetting } \\
\text { term }\end{array}$ & $\begin{array}{l}\text { With the forgetting } \\
\text { term }\end{array}$ \\
\hline $\begin{array}{l}\text { Maximum angle error } \\
\text { of hip joint }\end{array}$ & $1.88^{\circ} \pm 0.19$ & $3.06^{\circ} \pm 0.24$ & $1.96^{\circ} \pm 0.20$ & $3.02^{\circ} \pm 0.23$ \\
\hline $\begin{array}{l}\text { Maximum assistance } \\
\text { torque of hip joint }\end{array}$ & $115 \mathrm{Nm} \pm 3.1$ & $39 \mathrm{Nm} \pm 2.8$ & $117 \mathrm{Nm} \pm 2.9$ & $38 \mathrm{Nm} \pm 2.7$ \\
\hline
\end{tabular}

assistance torque according to subject's rehabilitation level. The robotic assistance depends on the forgetting factor value and the amount of subject's active participation.

\section{Conclusion}

In this article, a novel continuous seamless AAN control method based on SMAC framework was proposed to provide an adaptive seamless assistance torque according to subject's rehabilitation level for lower extremity rehabilitation robot. It was applied to a rehabilitation robot system operated at different gait rehabilitation stages and different rehabilitation training modes. Its performance was validated by 12 subjects with different rehabilitation levels, resulting in the following conclusions:
1. In the absence of forgetting term, even if subject has active motion ability, the robotic assistance torque does not decrease and the active effort of subject is inhibited.

2. With the forgetting term, the gait trajectory errors of all the subjects increase, and the assistance torques of the rehabilitation robot decrease. Moreover, the AAN controller can use a small trajectory deviation to decrease the robotic assistance torque in real time and achieve adaptive AAN control.

3. The robotic assistance torque depends on the forgetting factor and the level of subject's active participation.

4. The AAN controller used a small trajectory error to decrease the robotic assistance torque in real time can ensure a high trajectory tracking accuracy. Since the gait angle trajectory error data are 
continuous, the control method is no need for swing phase control and support phase control, separately. Thereby, it could be continuously adapted to different phases of the gait cycle. In addition, the control method can meet the gait rehabilitation training requirement of subjects at different rehabilitation levels. Thus, this could be applied to both the passive and active rehabilitation training modes to achieve continuous seamless adaptive AAN control.

5. Due to the limitations of experimental conditions, the therapeutic efficacy of the proposed continuous seamless adaptive AAN control method has not yet been measured.

The current results lay a groundwork for the test of subject's active role in improving the effectiveness of gait rehabilitation training. The proposed method can adapt to subject's active effort in real time, and it is no need to change parameters in different gait rehabilitation stages. It is expected that the research outlined in this article would provide guidance in solving continuous seamless AAN control related to different rehabilitation levels and different gait rehabilitation stages for improving the rehabilitation effect. This will be further verified through intensive user trials in the near future.

\section{Acknowledgements}

The authors gratefully acknowledge the financial support provided by National Natural Science Foundation of China under Grant NSFC (No. U1813212) and National Key R\&D Program of China (No. 2017 YFB1300505).

\section{Declaration of conflicting interests}

The author(s) declared no potential conflicts of interest with respect to the research, authorship, and/or publication of this article.

\section{Funding}

The author(s) disclosed receipt of the following financial support for the research, authorship, and/or publication of this article: This study was supported by National Natural Science Foundation of China under Grant NSFC (No. U1813212) and National Key R\&D Program of China (No. 2017YFB1300505).

\section{ORCID iD}

Gui Yin (D) https://orcid.org/0000-0002-1458-1868

\section{References}

1. Marchal-Crespo L and Reinkensmeyer DJ. Review of control strategies for robotic movement training after neurologic injury. J Neuro Eng Rehab 2009; 20: 1-15.
2. Hu J, Hou Z, Zhang F, et al. The lower limb rehabilitation robot and its interactive control method. Acta Autom Sin 2014; 40(11): 2377-2390.

3. Zhang X, Chen $\mathbf{J}$ and Yin G. An approach for humanrobot interactive control of lower limb rehabilitation robot based on sEMG perception. J Vibr Meas Diagn 2018; 38(4): 649-657.

4. Banala SK, Kim SH, Agrawal SK, et al. Robot assisted gait training with active leg exoskeleton. IEEE T Neural Syst Rehab Eng 2009; 17(1): 2-8.

5. Banala SK, Kim SH, Agrawal SK, et al. Novel gait adaptation and neuro motor training results using an active leg exoskeleton. IEEE/ASME T Mech 2010; 15(2): 216-225.

6. Hogan N, Krebs HI, Rohrer B, et al. Motions or muscles some behavioral factors underlying robotic assistance of motor recovery. J Rehab Res Dev 2006; 43(5): 605-618.

7. Cai LL, Fong AJ, Otoshi CK, et al. Implications of assist-as-needed robotic step training after a complete spinal cord injury on intrinsic strategies of motor learning. J Neuro Sci 2006; 26(41): 10564-10568.

8. Zhang Q, Zhang X, Yin G, et al. Design on subsection based mixed position controller for lower limb rehabilitation robot. In: Proceedings of the international conference on ubiquitous robots and ambient intelligence, Jeju, South Korea, 28 June-1 July 2017. New York: IEEE.

9. Cai LL, Fong AJ, Liang Y, et al. Assist-as-needed training paradigms for robotic rehabilitation of spinal cord injuries. In: Proceedings of the 2006 IEEE international conference robot and automation, Orlando, FL, 15-19 May 2006. New York: IEEE.

10. Michmizos KP and Krebs HI. Assist-as-needed in lower extremity robotic therapy for children with cerebral palsy. In: Proceedings of the 4th IEEE RAS/EMBS international conference on biomed robot bio-mechatronics, Rome, 24 27 June 2012. New York: IEEE.

11. Wang F, Barkana DE and Sarkar N. Impact of visual error augmentation when integrated with assist-as-needed training method in robot assisted rehabilitation. IEEE T Neural Syst Rehab Eng 2010; 18(5): 571-579.

12. Hu J, Hou Z, Zhang F, et al. Training strategies for a lower limb rehabilitation robot based on impedance control. In: Proceedings of the 34th annual international conference of the IEEE EMBS, San Diego, CA, 28 August-1 September 2012. New York: IEEE.

13. Hussain S, Xie SQ and Jamwal PK. Adaptive impedance control of a robotic orthosis for gait rehabilitation. IEEE T Cybern 2013; 43(3): 1025-1034.

14. Lee J, Chang PH, et al. Relative impedance control for dual-arm robots performing asymmetric bimanual tasks. IEEE T Ind Electr 2014; 61(7): 3786-3796.

15. Duschau-Wicke A, Caprez A, Riener R, et al. Path control: a method for patient-cooperative robot aided gait rehabilitation. IEEE T Neural Syst Rehab Eng 2010; 18(1): 38-48.

16. Toshio $\mathrm{T}$ and Yoshiyuki $\mathrm{T}$. Tracking control properties of human-robotic systems based on impedance control. IEEE T Syst Man Cybern: Part A: Syst Human 2005; 35(4): 523-535.

17. He W and Dong Y. Adaptive fuzzy neural network control for a constrained robot using impedance learning. IEEE T Neural Netw Learn Syst 2018; 29(4): 1174-1186. 
18. Pérez-Ibarra JC, Siqueira AG and Krebs HI. Assist-asneeded ankle rehabilitation based on adaptive impedance control. In: Proceedings of the IEEE international conference on rehabilitation robotics, Singapore, 11-14 August 2015. New York: IEEE.

19. Yu H, Huang S, Chen G, et al. Human-robot interaction control of rehabilitation robots with series elastic actuators. IEEE T Robot 2015; 31(5): 1089-1100.

20. Veneman JF, Kruidhof R, Hekman EEG, et al. Design and evaluation of the LOPES exoskeleton robot for interactive gait rehabilitation. IEEE T Neural Syst Rehab Eng 2007; 15(3): 379-386.

21. Chen G, Ye J, Duan LH, et al. Adaptive control strategy for gait rehabilitation robot to assist-when-needed. In: Proceedings of the IEEE international conference on realtime computing and robotics, Kandima, Maldives, 1-5 August 2018. New York: IEEE.

22. Cao JH, Xie SQ, Das R, et al. Control strategies for effective robot assisted gait rehabilitation: the state of art and future prospects. Med Eng Phys 2014; 36: 1556-1565.

23. Teramae T, Noda T and Morimoto J. EMG-based model predictive control for physical human-robot interaction: application for assist-as-needed control. IEEE Robot Autom Lett 2018; 3(1): 210-217.

24. Akim K, Shahid H, Askhat S, et al. Brain-computer interface and assist-as-needed model for upper limb robotic arm. Adv Mech Eng 2019; 11(9): 1-9.

25. Zhang T, Wang X, Philip Chen CL, et al. GCB-net: graph convolutional broad network and its application in emotion recognition. J Latex Class Files 2019; 14(8): 110.

26. Zelinsky A. Robot suit hybrid assistive limb. IEEE Robot Autom Mag 2009; 16(4): 98-102.

27. Wolbrecht ET, Chan V, Bobrow JE, et al. Optimizing compliant, model based robotic assistance to promote neurorehabilitation. IEEE T Neural Syst Rehab Eng 2008; 16(3): 286-297.

28. Hussain S and Xie SQ. Design and assist-as-needed control of an intrinsically compliant robotic orthosis for gait rehabilitation. PhD Thesis, The University of Auckland, Auckland, New Zealand, 2012.

29. Wu Q, Wang X, Chen B, et al. Development of a minimal-intervention-based admittance control strategy for upper extremity rehabilitation exoskeleton. IEEE T Syst Man Cybern: Syst 2018; 48(6): 1005-1016.
30. Yin G, Zhang X, Chen J, et al. Trajectory tracking adaptive control of the lower limb rehabilitation robot with model uncertainty. J Electr Meas Instrument 2016; 30(11): $1750-1757$.

31. Liu C, Wen G, Sedaghati R, et al. Neural-network-based sliding-mode control of an uncertain robot using dynamic model approximated switching gain. IEEE T Cybern. Epub ahead of print 18 March 2020. DOI: 10.1109/ TCYB.2020.2978003.

32. Zhang T, Chen $\mathrm{P}, \mathrm{Hu} \mathrm{B}$, et al. Design of highly nonlinear substitution boxes based on I-Ching operators. IEEE T Cybern 2018; 48(12): 3349-3358.

33. Yin H, Yu D, Chen Y-H, et al. Nash-game-oriented optimal design in controlling fuzzy dynamical systems. IEEE T Fuzzy Syst 2019; 27(8): 1659-1673.

34. Yin $\mathrm{H}$, Huang $\mathrm{J}$, Chen $\mathrm{Y}-\mathrm{H}$, et al. Tackling mismatched uncertainty in robust constraint-following control of under-actuated systems. Inform Sci 2020; 520: 337-352.

35. Yang $\mathrm{K}$, Zhang X, Yin G, et al. Design of rehabilitation training robot for lower limb of human body. Mach Electr 2018; 36(3): 55-60.

36. Walid H, Samer M, Rifai H, et al. EMG based approach for wearer-centered control of a knee joint actuated orthosis. In: Proceedings of the IEEE/RSJ international conference on intelligent robots and systems, Tokyo, Japan, 3-7 November 2013. New York: IEEE.

37. Yin YH, Fan YJ and Xu LD. EMG and EPP-integrated human-machine interface between the paralyzed and rehabilitation exoskeleton. IEEE T Inform Tech Biomed 2012; 16(4): 542-549.

38. Spong MW and Midyasagar M. Robot dynamics and control. New York: Wiley, 1989, pp. 265-267.

39. Slotine JJE and Li W. Applied nonlinear control. Beijing, China: Mechanical Industry Press, 2006, pp. 168-213.

40. Lü H, Shangguan W-B and Yu D. Uncertainty quantification of squeal instability under two fuzzy-interval cases. Fuzzy Sets Syst 2017; 328: 70-82.

41. Lü H, Shangguan W-B, Yu D, et al. An improved method for fuzzy-interval uncertainty analysis and its application in brake instability study. Comput Method Appl Mech Eng 2018; 342: 142-160.

42. Riener R, Lunenburger L, Jezernik S, et al. Patient-cooperative strategies for robot-aided treadmill training: first experimental results. IEEE T Neural Syst Rehab Eng 2005; 13: 380-394. 